\title{
Activity in the human amygdala corresponds to early, rather than late period autonomic responses to a signal for shock
}

\author{
Dominic T. Cheng, ${ }^{1,3}$ Jennifer Richards, ${ }^{1}$ and Fred J. Helmstetter ${ }^{1,2,4}$ \\ ${ }^{1}$ Department of Psychology, University of Wisconsin-Milwaukee, Milwaukee, Wisconsin 53201, USA; ${ }^{2}$ Medical College \\ of Wisconsin, Milwaukee, Wisconsin 53226, USA
}

\begin{abstract}
Laboratory animal and human subject studies report that the amygdala is a critical brain structure that supports the acquisition and expression of conditional fear. Recent functional neuroimaging studies in humans have reported that activity in this region is closely related to the behavioral expression of conditional skin conductance responses (SCR). However, SCR waveforms following conditional stimulus (CS) presentation contain both early period and late period responses that may differ with respect to underlying central processes. It is not known whether amygdala activity corresponds to the expression of early conditonal responses (CRs) that occur shortly following CS onset or late CRs that closely precede UCS onset. The present study used event-related functional magnetic resonance imaging and concurrent skin conductance measurements to determine whether amygdala activity is more closely related to the expression of early or late period CRs. Increased amygdala activity was detected during the formation of early, but not late period CRs. Additionally, this pattern of amygdala activity did not dissipate, but persisted into late stages of the experiment. These findings are consistent with the idea that amygdala responding is critically involved in the generation of CRs formed shortly following CS onset.
\end{abstract}

Pavlovian fear conditioning has been widely used as a model system to study the neural mechanisms of associative learning and memory. In this form of learning, a conditional stimulus (CS) is paired with a noxious, unconditional stimulus (UCS) such as electric shock. Following repeated pairings of the CS and UCS, the CS alone begins to elicit behavioral conditional responses (CR). Expression of the CR is taken as evidence that an animal has learned an association between the CS and UCS. The neural pathways underlying this form of learning have been studied extensively using both laboratory animals and human subjects (Davis 2000; LeDoux 2000; Phelps and LeDoux 2005).

Human lesion and neuroimaging findings have supported the idea that the amygdala, a key neural component of the fear conditioning circuit, is critically involved in the expression of learned autonomic fear responses (Bechara et al. 1995; Büchel et al. 1998; LaBar et al. 1995, 1998; Phelps et al. 2001; Cheng et al. 2003, 2006; Knight et al. 2005). During fear conditioning, patients with amygdala lesions failed to demonstrate conditional skin conductance responses (SCR), while their declarative knowledge of the conditioning procedures remained intact (Bechara et al. 1995; LaBar et al. 1998). It is unlikely that damage to this region is generally involved in the control of SCR because patients with amygdala lesions show normal electrodermal responding to auditory and visual stimuli (Tranel and Damasio 1989, 1993). Instead, these results suggest that damage to the amygdala likely produced deficits in the ability to generate conditional SCRs.

Consistent with this idea, functional magnetic resonance imaging (fMRI) studies have also demonstrated a relationship between human amygdala activity and the autonomic expres-

\footnotetext{
${ }^{3}$ Present address: Johns Hopkins University School of Medicine, Baltimore, Maryland 21205, USA.

${ }^{4}$ Corresponding author.

E-mail fjh@uwm.edu; fax (414) 229-5219.

Article is online at http://www.learnmem.org/cgi/doi/10.1101/lm.632007.
}

sion of conditional fear (Büchel et al. 1998; LaBar et al. 1998; Phelps et al. 2001; Cheng et al. 2003, 2006; Knight et al. 2005). Previous work from our laboratory showed that activity within the human amygdala closely resembled waveforms generated from the subject's conditional SCRs (Cheng et al. 2003). Furthermore, increased amygdala activity has been detected only on trials that both signaled shock and produced a significant conditional SCR, suggesting that responding in this structure may not exclusively reflect learning stimulus associations, but also reflect the expression of conditional fear responses (Knight et al. 2005; Cheng et al. 2006).

Multiple, distinct conditional fear responses have been identified upon a closer examination of the SCR waveform (Leonard and Winokur 1963; Prokasy and Ebel 1967; Wolter and Lachnit 1993). Skin conductance responses during the CS interval can be separated into two periods: an early, first interval response (FIR) and a late, second interval response (SIR). The FIR occurs shortly following CS onset and the SIR is defined as a response that occurs closer to when the UCS would be presented. The FIR is also considered an orienting response to the CS and may reflect more attentional than associative processes (Prokasy and Raskin 1973). In contrast, the SIR is an anticipatory response to the UCS and is a better representation of the degree of arousal as a function of the subject learning the relation between the CS and the UCS. Since both FIR and SIR may reflect separate psychological processes, several behavioral studies have been performed to test whether these two response types can be conditioned independently (Leonard and Winokur 1963; Prokasy and Ebel 1967; Wolter and Lachnit 1993).

Wolter and Lachnit (1993) found a linear relationship between the magnitude of subjects' SCR and UCS intensity. Higher SCR amplitudes were observed during both the FIR and SIR periods to increasingly aversive UCSs, although this relationship was significantly stronger for SIR. Leonard and Winokur (1963) presented a tone that was paired with shock and another tone that was explicitly unpaired. The number of FIRs elicited by the 
two tones was similar, but the number of SIRs was significantly greater on paired than on unpaired trials. Conversely, Prokasy and Ebel (1967) found that paired tone-shock presentations elicited significantly higher magnitude FIR relative to unpaired tone-shock presentations, but no differences were observed during the SIR period. One possible explanation for this discrepancy is that Leonard and Winokur (1963) compared the number of responses during each interval, whereas Prokasy and Ebel (1967) compared the magnitude of responses. Nevertheless, these two very early studies suggest that conditional FIRs and conditional SIRs may be independent of each other.

SCR learning-related changes can be manifested through early and late period autonomic responses (Leonard and Winokur 1963; Prokasy and Ebel 1967; Wolter and Lachnit 1993). Since these distinct responses may reflect separate psychological processes during fear conditioning, the neural mechanisms underlying these response types may also be different. Although current data suggest that the expression of conditional SCRs may be a function of human amygdala activity (Büchel et al. 1998; LaBar et al. 1998; Phelps et al. 2001; Cheng et al. 2003, 2006; Knight et al. 2005), it is currently not known whether this activity corresponds to early or late period autonomic responses to a signal for shock.

The present study expanded on the general approach of using learned autonomic fear responses to map neural activity (Cheng et al. 2003, 2006; Knight et al. 2005). The current analysis technique explored amygdala activity associated with different trial types based on first (early) and second (late) interval skin conductance responses. A CS period of $20 \mathrm{sec}$ was used to accommodate analyses of multiple CRs, and functional images were restricted to the amygdala in order to achieve greater temporal resolution.

\section{Results}

\section{Shock expectancy and SCR}

A repeated measures ANOVA was performed on UCS Expectancy data for CS+ and CS - trials over the training session. Differential responding to CS presentations developed across time as demonstrated by a significant main effect for CS $\left(F_{(1,11)}=57.75\right.$, $P<0.05)$, main effect for trial $\left(F_{(1,11)}=15.96, P<0.05\right)$, and a significant CS $\times$ Trial interaction $\left(F_{(1,11)}=67.58, P<0.05\right)$. Furthermore, post-hoc comparisons revealed no differences between CS presentations on trial $1(t=0.16, P>0.05)$, but significantly higher expectancy ratings during CS+ presentations by trial 2 $(t=2.67, P<0.05)$, suggesting rapid acquisition of stimulus contingencies (see Fig. 1A).
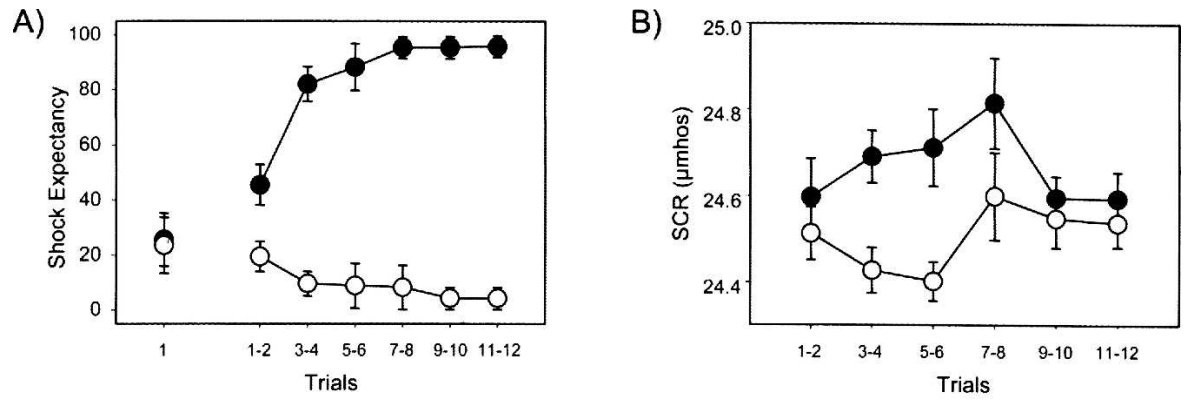

\section{- $\mathrm{CS}+\mathrm{CS}$.}

Figure 1. Behavioral data across $12 \mathrm{CS}+$ and $12 \mathrm{CS}-$ trials. $(A)$ Averaged explicit responses to $\mathrm{CS}+$ and CS - trials. Subjects demonstrated rapid acquisition of the CS-UCS relationship. (B) Averaged SCR to CS+ and CS - trials. CS+ trials elicited significantly higher magnitude SCRs relative to CS - trials, demonstrating implicit discrimination in subjects.
A repeated measures ANOVA was also performed on SCR data for CS+ and CS - trials over the training session. Differential responding between CS presentations were observed as evidenced by a significant main effect for CS $\left(F_{(1,11)}=10.40\right.$, $P<0.05)$. Post-hoc comparisons indicated increased SCR amplitude to CS+ presentations on trials 3 and $4(t=2.66, P<0.05)$ and trials 5 and $6(t=3.06, P<0.05)$ (see Fig. 1B). Finally, averaged SCR waveforms during the first half of the experiment for both early period CR and late period CR trial types are presented at the bottom of Figure 2.

\section{fMRI}

To determine whether amygdala activity was related more to CRs formed in the first interval or more to CRs formed in the second interval of CS+ presentations, contrasts between trials with early period CRs and trials with late period CRs were performed. This analysis yielded a cluster of activation in the right amygdala (Talairach coordinates: $13-7-12 ; 172 \mu \mathrm{L}$ ). Significant differential amygdala activity between these two trial types was detected and characterized by increased responding during early period CR trials and a deactivation during late period CR trials (see Fig. 2). Furthermore, these response patterns were consistent in both the first and second half of the experiment (see Fig. 3).

To investigate the possibility that amygdala responding may be related to nonassociative SCRs (e.g., unconditional responses), contrasts between post-CS+ and post-CS - periods were also performed. Differential amygdala responding was not detected in this comparison. Consistent with previous findings from our laboratory (Cheng et al. 2006), analyses of CS - trials (including both response and nonresponse trial types) revealed little to no activation throughout the experiment (\% AUC: $0.06 \pm 0.10)$.

\section{Discussion}

Several independent laboratories demonstrated that human amygdala activity was closely related to the expression of conditional SCRs (Büchel et al. 1998; LaBar et al. 1998; Phelps et al. 2001; Cheng et al. 2003, 2006; Knight et al. 2005). Results from the current study expand on this idea by showing that increased amygdala activity was more closely related to early period, rather than late period, autonomic responses to a signal for shock. While early CRs were associated with increased amygdala activity, late CRs were related to decreased amygdala activity (see Fig. 2). Furthermore, this pattern of responding also appeared to be consistent in both the first and second halves of the experiment (see Fig. 3).

Early behavioral responses to CS onset are generally considered orienting responses and may reflect more attentional than associative processes, while late responses are more anticipatory of the UCS and are a better representation of the subject learning the relation between the CS and the UCS. However, one important index of an organism's encoding of the predictive relationship between the CS and UCS is the preservation or maintenance of these responses as a function of learning (Stern and Walrath 1977; Holland 1984). This account predicts that maintenance of the early SCR amplitude due to CS-UCS pairings could also be considered CRs. A closer examination of early response trials (bottom of Fig. 2) will show that this initial response was maintained over the 


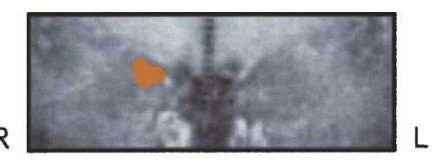

A)

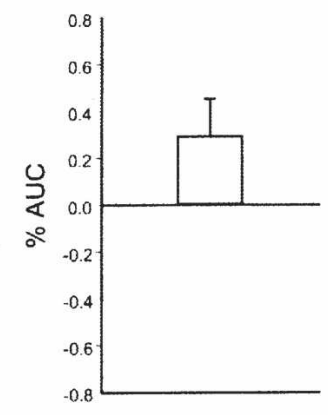

C)

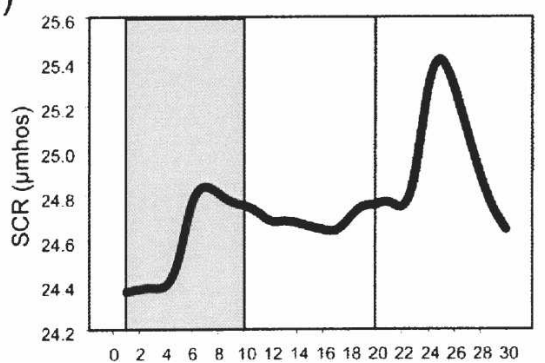

B) Late Period CRs

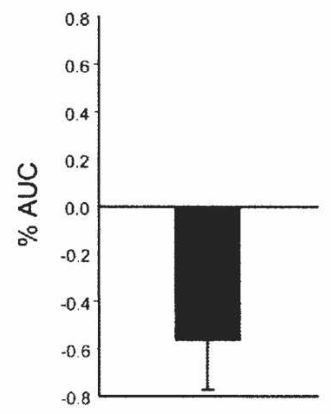

D)

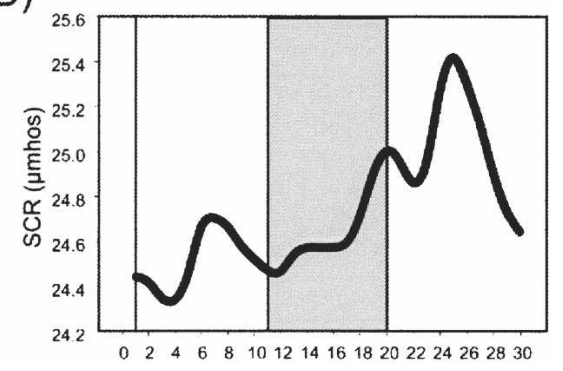

Figure 2. Imaging and behavioral data for early and late period CRs during the first half of the experiment. (Top) Coronal close-up of a contrast between early and late CRs revealed a cluster of activation in the right amygdala (Talairach coordinates: $13-7-12 ; 172 \mu \mathrm{L}$ ). (A) Increased amygdala activity was detected during early period CRs. (B) Decreased amygdala activity was detected during late period CRs. (C) Averaged SCR waveform during trials that elicited early CRs. $(D)$ Averaged SCR waveform during trials that elicited late CRs.

course of the CS period. In conjunction with the imaging data, these results indicate that early preserved CRs over the CS period are one reliable predictor of amygdala activity. This pattern of activity is also consistent with both neuroimaging (Phelps et al. 2001) and electrophysiological (Quirk et al. 1997) data that show early transient amygdala activity. Phelps et al. (2001) showed significant, transient amygdala activity during the first half of an 18 -sec CS in an "instructed" fear procedure, and Quirk et al. (1997) showed amygdala cells primarily respond only in the first few milliseconds of CS presentations.

Late CRs formed closer to UCS onset produced substantial decreased amygdala activity. Several factors may have contributed to this pattern of activation. First, this deactivation may be related to a post-stimulus undershoot, which is commonly observed following an initial peak in the blood oxygenation leveldependent (BOLD) signal. Differences between blood flow and volume following neuronal activation attenuate the BOLD signal and may account for this below-baseline activity (Mandeville et al. 1999). Second, deactivation in the amygdala has been observed during noxious stimulation in several studies (Derbyshire et al. 1997; Becerra et al. 1999; Petrovic et al. 2004). In a positron emission tomography (PET) study by Petrovic et al. (2004), the authors attributed deactivation in the amygdala to subjects developing strategies to attenuate responses associated with the pain. Third, decreases in amygdala responding have been reported during the presence of long stimuli durations (Poellinger et al. 2001). In this study, long duration olfactory stimuli (60 sec) produced a pattern of amygdala responding that was remarkably similar to the pattern observed in the current study: a short, transient increase in amygdala activity followed by a decrease below baseline. The combined factors of a post-stimulus undershoot, a painful stimulus, and a long CS duration may have played a role in the pattern of deactivation seen during late period autonomic responses to a signal for shock.

Amygdala responding reported in the current study cannot be attributed to general SCR fluctuations and arousal for several reasons. Little to no amygdala activity was seen on CS - trials that elicited a positive SCR during both early and late periods of the stimulus. Also, contrasts designed to outline regions important for UCR generation (post-CS+ vs. post-CS - ) did not reveal differential responding in the amygdala. Furthermore, Figure 4 shows a similar topography of responding between early and late

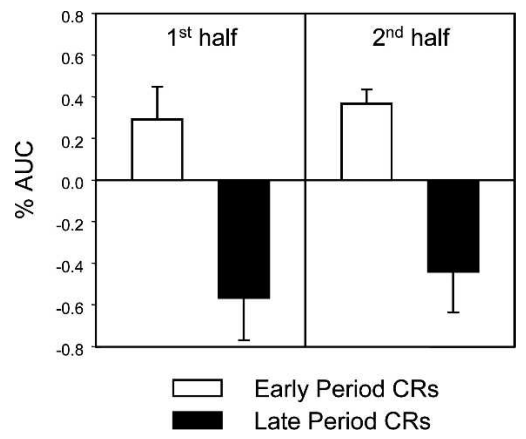

Figure 3. Comparison of amygdala responding during the first and second half of the experiment. White bars indicate amygdala activity during trials that elicited early CRs, while black bars denote amygdala responding during late CRs. 


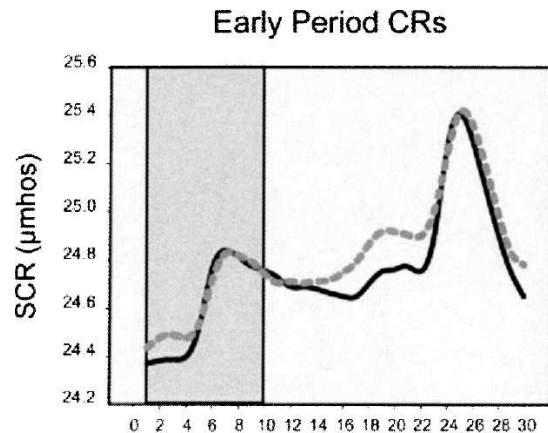

Early Period CRs

$\begin{array}{llllllllll}0 & 2 & 4 & 8 & 8 & 101214 & 16182022 & 24262830\end{array}$

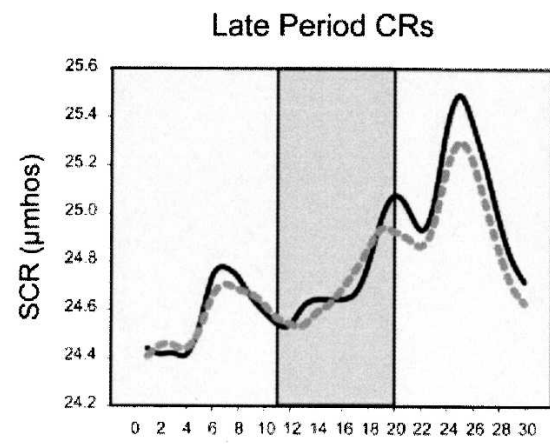

$1^{\text {st }}$ half
$=m \quad 2^{\text {nd }}$ half

Figure 4. A similar topography of conditional SCRs was observed between the first and second half of the study. Solid lines signify responding during the first half of the study, and dotted lines refer to the second half of the study. Increased amygdala responding was only detected during early period responses (shaded area of left graph) but not during late period responses (shaded area of right graph).

period response trials (shaded areas in graphs) throughout the experiment. If increased amygdala activity was simply associated with a positive SCR, it should have been detected during late period responses as well. Finally, results from several functional neuroimaging and amygdala lesion studies are consistent with the idea that amygdala activity is independent of the production of general SCRs (Tranel and Damasio 1989, 1993; Critchley et al. 2000; Patterson et al. 2002; Knight et al. 2005). Lesion data have shown that amygdala patients can demonstrate intact SCR patterns (Tranel and Damasio 1989, 1993), and fMRI studies failed to detect amygdala activity during the formation of nonspecific SCRs (Critchley et al. 2000; Patterson et al. 2002; Knight et al. 2005). These data support the idea that the amygdala is not necessary for general SCR fluctuations and arousal.

Overall, the current results are in strong agreement with previous fear-conditioning studies that report increased amygdala activity corresponding to the expression of conditional SCRs (Büchel et al. 1998; LaBar et al. 1998; Phelps et al. 2001; Cheng et al. 2003, 2006; Knight et al. 2005). In particular, Phelps et al. (2001), in an "instructed" fear procedure, found significant amygdala activity during the first half of an 18-sec CS, which was attenuated during the second half. This early activation was significantly correlated with subject's electrodermal responding, suggesting a relationship between amygdala responding and learning-related changes in SCR. However, a distinction between early and late period autonomic responses was not made. Although Knight et al. (2005) behaviorally distinguished between first and second interval SCRs, they did not report amygdala activity uniquely associated with either type of response. Several methodological differences may account for this discrepancy. Their CS duration was $10 \mathrm{sec}$, while the present study used a 20 -sec CS. We felt that extending the duration of the CS period would increase our ability to distinguish first- and secondinterval responses. Furthermore, Knight et al. (2005) used wholebrain coverage, limiting them to a temporal resolution of $\mathrm{TR}=2$, while the current study restricted coverage to the amygdala, allowing images to be collected at a resolution of $\mathrm{TR}=1$.

Detecting increased amygdala responding during the second half of the experiment is an important and unique finding. Many fear-conditioning studies have reported a transient role for the amygdala, and that activity within this region habituates over time (Büchel et al. 1998; LaBar et al. 1998; Phelps et al. 2001; Cheng et al. 2003, 2006). If activity in the amygdala is uniquely involved in the expression of conditional SCRs, then it stands to trials that correspond to the expression of early conditional SCRs. In conclusion, the present study was designed to expand on previous work, which argued that human amygdala activity was closely related to the production of conditional SCRs. By extending the CS duration, we were able to distinguish between early and late CRs. Results demonstrated that significant amygdala activity corresponded to trials that elicited early, but not late period conditional SCRs. Critically, these early electrodermal responses were maintained throughout the CS duration and served as an index of the subject learning the CS-UCS association. Furthermore, this pattern of amygdala activity and electrodermal responding continued throughout the second half of the experiment, suggesting that amygdala activity can persist throughout late stages of experiments, but only on trials that are capable of eliciting early conditional SCRs.

\section{Materials and Methods}

\section{Subjects}

Twelve healthy, right-handed subjects (six males) aged $20.4 \pm 0.5$ yr were recruited. All procedures were approved by the Institutional Review Boards for human subject research at both the Medical College of Wisconsin and the University of Wisconsin-Milwaukee.

\section{Apparatus}

\section{MRI}

Functional imaging was performed on a 3.0 Tesla/60 Bruker Biospec scanner using a multislice gradient-echo echoplanar pulse sequence. Four contiguous 3-mm coronal slices centered around the amygdala were collected $(\mathrm{TR}=1000 \mathrm{msec}, \mathrm{TE}=27.2 \mathrm{msec}$, FOV $=24 \mathrm{~cm}$, Flip Angle $=90^{\circ}$ ) in a series of 1230 sequential images (for a total of $1230 \mathrm{sec}$ ). High-resolution anatomical images were obtained with a 3D multi-planar inversion recovery (MPIR) gradient-echo imaging sequence to serve as an anatomical map over which functional images were superimposed.

\section{Electrical stimulus}

The UCS was electrical stimulation $(0.5 \mathrm{sec})$ presented via a custom-made AC $(60 \mathrm{~Hz})$ source through two aluminum surface electrodes ( $2 \mathrm{~cm}$ diameter) positioned over the right tibial nerve above the right medial malleolus. Subjects were asked to individually set the intensity of the electrical stimulus. Prior to training, practice trials were given in which subjects rated the level of electrical stimulation on a scale from 0 to $5(0=$ no sensation, 
5 = painful, but tolerable). Gradual increases in intensity, interspersed with two declinations, were given until the stimulus intensity that elicited a subjective report of 5 was achieved. The maximum possible current used for the UCS was $7.35 \mathrm{~mA}$.

\section{Skin conductance response}

Two surface cup electrodes (silver/silver chloride, 1-cm diameter, Beckman Instruments) filled with electrolyte gel (Teca Corp.) was attached $2 \mathrm{~cm}$ apart to the sole of the subject's left foot. Skin conductance data was digitized and stored continuously at 250 $\mathrm{Hz}$ using Asyst software (Version 3.10).

\section{UCS expectancy}

A custom-made rotary dial was used to monitor the subject's expectancy of receiving electrical stimulation. The dial controlled a ratings bar presented at the bottom of the visual display. Real-time feedback of the subject's dial position was displayed throughout the experiment. Ratings were made on a continuous scale ranging from 0 to 100 , where 0 reflected the expectation that shock definitely would not be presented and 100 reflected the expectation that shock definitely would be presented. A rating of 50 indicated that subjects were uncertain as to whether the shock would be presented. Subjects were instructed to continuously update their rating to accurately reflect their current shock expectancy. The dial was attached to the right thigh with a Velcro strap and subjects were instructed to manipulate the dial with their right hand.

\section{Visual stimuli}

Twenty-four trials of two conditional stimuli (12 CS+, 12 CS -) were presented (20-sec duration, 50-sec ITI) during the experiment. Conditional stimuli were a single white square or triangle and were counter-balanced and presented in a pseudo-random order such that no more than two trials of the same CS were consecutively presented. CS+ trials coterminated with UCS presentations and CS - trials were presented alone.

\section{Data analysis}

\section{Trial classification}

Prior to imaging analyses, CS trials were divided into early and late period response trial types based on an individual subject's SCR. Early period responses were defined as responses that occurred during the first $10 \mathrm{sec}$ of the CS and late period responses were defined as responses that occurred during the last $10 \mathrm{sec}$. A response was classified as a CR if the range between the maximum and minimum response was greater than three times the standard deviation of the mean of the baseline period (10 sec prior to CS) (Cheng et al. 2006). Importantly, a late period response was not always preceded by an early period response and vice versa. This variability addresses the problem of collinearity during imaging analyses and contributes during the estimation of the impulse response function (IRF) related to both early and late period skin conductance responses.

\section{Imaging data}

Functional MRI time series data were analyzed with the 3D Deconvolution program from the AFNI software package (Cox 1996), which used the measured blood-oxygenated level dependent (BOLD) response and input reference functions representing the temporal location of the trial types to estimate the IRF evoked by each trial type. The percentage of area under the IRF curve (\%AUC) was used as a measure of response strength.

Functional and anatomical images for each subject were transformed into stereotaxic coordinate space relative to the line between the anterior and posterior commissures. To compensate for anatomical variability between subjects, a 4-mm full-widthat-half-maximum Gaussian blur was applied to each subject's functional data set following transformation to common coordinate space. Amygdala responses associated with each trial type were compared using parametric statistics. Volumes of active tissue meeting an inclusion criteria of $P<0.01$ were used in a functional region of interest (ROI) analysis. Increased responding during the expression of early period CRs would suggest amygdala activity was more closely related to orienting conditioned responses, while greater responding during the expression of late period CRs would indicate that this region was more important for anticipatory fear responses to the UCS.

\section{Acknowledgments}

This work was supported by the National Institute of Mental Health Grant (MH060668) awarded to F.J.H. and a Bill and Melinda Gates Foundation Scholarship awarded to D.T.C.

\section{References}

Becerra, L.R., Breiter, H.C., Stojanovic, M., Fishman, S., Edwards, A., Comite, A.R., Gonzalez, R.G., and Borsook, D. 1999. Human brain activation under controlled thermal stimulation and habituation to noxious heat: An fMRI study. Magn. Reson. Med. 41: 1044-1057.

Bechara, A., Tranel, D., Damasio, H., Adolphs, R., Rockland, C., and Damasio, A.R. 1995. Double dissociation of conditioning and declarative knowledge relative to the amygdala and hippocampus in humans. Science 269: 1115-1118.

Büchel, C., Morris, J., Dolan, R.J., and Friston, K.J. 1998. Brain systems mediating aversive conditioning: An event-related fMRI study. Neuron 20: 947-957.

Cheng, D.T., Knight, D.C., Smith, C.N., Stein, E.A., and Helmstetter, F.J. 2003. Functional MRI of human amygdala activity during Pavlovian fear conditioning: Stimulus processing versus response expression. Behav. Neurosci. 117: 3-10.

Cheng, D.T., Knight, D.C., Smith, C.N., and Helmstetter, F.J. 2006. Human amygdala activity during the expression of fear responses. Behav. Neurosci. 120: 1187-1195.

Cox, R.W. 1996. AFNI: Software for the analysis and visualization of functional magnetic resonance images. Comput. Biomed. Res. 29: $162-173$.

Critchley, H.D., Elliot, R., Mathias, C.J., and Dolan, R.J. 2000. Neural activity relating to generation and representation of galvanic skin conductance responses: A functional magnetic resonance imaging study. J. Neurosci. 20: 3033-3040.

Davis, M. 2000 The role of the amygdala in conditioned and unconditioned fear and anxiety. In The amygdala: A functional analysis (ed. J Aggleton), pp. 213-288. Oxford University Press, New York.

Derbyshire, S.W., Jones, A.K., Gyulai, F., Clark, S., Townsend, D., and Firestone, L.L. 1997. Pain processing during three levels of noxious stimulation produces differential patterns of central activity. Pain 73: $431-445$.

Holland, P.C. 1984. The origins of Pavlovian conditioned behavior. In The psychology of learning and motivation (ed. G. Bower), pp. 129-173. Academic Press, Orlando, FL.

Knight, D.C., Nguyen, H.T., and Bandettini, P.A. 2005. The role of the human amygdala in the production of conditioned fear responses. Neuroimage 26: 1193-1200.

LaBar, K.S., LeDoux, J.E., Spencer, D.D., and Phelps, E.A. 1995. Impaired fear conditioning following unilateral temporal lobectomy in humans. J. Neurosci. 15: 6846-6855.

LaBar, K.S., Gatenby, C., Gore, J.C., LeDoux, J.E., and Phelps, E.A. 1998. Human amygdala activation during conditioned fear acquisition and extinction: A mixed trial fMRI study. Neuron 20: 937-945.

LeDoux, J.E. 2000. Emotion circuits in the brain. Annu. Rev. Neurosci. 23: $155-184$.

Leonard, C. and Winokur, G. 1963. Conditioning versus sensitization in the galvanic skin response. J. Comp. Physiol. Psychol. 56: 169-170.

Mandeville, J.B., Marota, J.J., Ayata, C., Moskowitz, M.A., Weisskoff, R.M., and Rosen, B.R. 1999. MRI measurement of the temporal evolution of relative $\mathrm{CMRO}_{2}$ during the rat forepaw stimulation. Magn. Reson. Med. 42: 944-951.

Patterson II, J.C., Ungerleider, L.G., and Bandettini, P.A. 2002. Task-independent functional brain activity correlation with skin conductance changes: An fMRI study. Neuroimage 17: 1797-1806.

Petrovic, P., Carlsson, K., Petersson, K.M., Hansson, P., and Ingvar, M. 2004. Context-dependent deactivation of the amygdala during pain. J. Cogn. Neurosci. 16: 1289-1301.

Phelps, E.A. and LeDoux, J.E. 2005. Contributions of the amygdala to emotion processing: From animal models to human behavior. Neuron 48: 175-187. 
Phelps, E.A., O'Connor, K.J., Gatenby, C., Gore, J.C., Grillon, C., and Davis, M. 2001. Activation of the left amygdala to a cognitive representation of fear. Nat. Neurosci. 4: 437-441.

Poellinger, A., Thomas, R., Lio, P., Lee, A., Makris, N., Rosen, B.R., and Kwong, K.K. 2001. Activation and habituation in olfaction-an fMRI study. Neuroimage 13: 547-560.

Prokasy, W.F. and Ebel, H.C. 1967. Three components of the classically conditioned GSR in human subjects. J. Exp. Psychol. 73: 247-256.

Prokasy, W.F. and Raskin, D.C. 1973. Electrodermal activity in psychological research. Academic Press, New York.

Quirk, G.J., Armony, J.L., and LeDoux, J.E. 1997. Fear conditioning enhances different temporal components of tone-evoked spike trains in auditory cortex and lateral amygdala. Neuron 19: 613624.

Stern, J.A. and Walrath, L.C. 1977. Orienting responses and conditioning of electrodermal responses. Psychophysiology 14: $334-342$.

Tranel, D. and Damasio, H. 1989. Intact electrodermal skin conductance responses after bilateral amygdala damage. Neuropsychologia 27: 381-390.

Tranel, D. and Damasio, A.R. 1993. The covert learning of affective valence does not require structures in hippocampal system or amygdala. J. Cogn. Neurosci. 5: 79-88.

Wolter, J. and Lachnit, H. 1993. Are anticipatory first and second interval skin conductance responses indicators of predicted aversiveness? Integr. Physiol. Behav. Sci. 28: 163-166.

Received May 17, 2007; accepted in revised form May 25, 2007. 


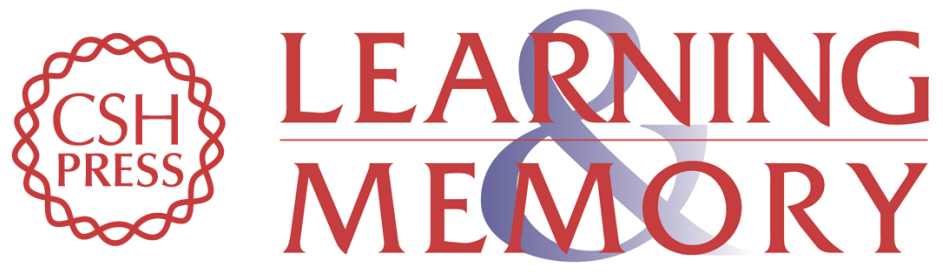

\section{Activity in the human amygdala corresponds to early, rather than late period autonomic responses to a signal for shock}

Dominic T. Cheng, Jennifer Richards and Fred J. Helmstetter

Learn. Mem. 2007, 14:

Access the most recent version at doi:10.1101//m.632007

References This article cites 25 articles, 3 of which can be accessed free at:

http://learnmem.cshlp.org/content/14/7/485.full.html\#ref-list-1

License

Email Alerting Receive free email alerts when new articles cite this article - sign up in the box at the Service top right corner of the article or click here. 